\title{
STDP: spiking, timing, rates and beyond
}

\section{Leon N Cooper*}

Department of Physics, Institute for Brain and Neural Systems, Brown University, Providence, RI, USA

*Correspondence: inc@brown.edu

Our view of the world has changed dramatically since it was realized in the early 1970 s that networks of neurons can form mappings that are associative, content addressable and relatively invulnerable to the loss of individual neurons or synapses - thus potential candidates for memory storage in the animal brain (Anderson et al., 1972). But how, we asked ourselves, could such mappings be constructed in networks of neurons? That is, how could the values of vast numbers of synapses be adjusted to obtain a mapping that corresponds to an appropriate memory?

One possibility was that synaptic modification followed the famous Hebbian rule: "When an axon in cell A is near enough to excite cell B and repeatedly and persistently takes part in firing it, some growth process or metabolic change takes place in one or both cells such that A's efficiency in firing B is increased" (Hebb, 1949). Or, "Neurons that fire together wire together." In restricted circumstances this gave a mapping with some of the properties of memory.

But, though Hebb had proposed this idea in 1949, it had hardly become fashionable in the biological community. I recall giving talks on this subject, being greeted with condescending smiles and, perhaps, a pat on the head from established neurophysiologists: "You're very clever young man, but what shred of evidence do you have that synapses modify?" I remember, in particular, an extended conversation with a famous neuroscientist in the late 70s: "We have no evidence whatsoever for Hebbian modification in the ugly little sea snail that I am studying," he would say. (The evidence has since been obtained, Lin and Glanzman, 1994.) I remember, in exasperation, suggesting "Well perhaps that's the difference between an ugly little sea snail and a good looking tenured professor at a major university."

It was evident, of course, that Hebbian modification could be only part of the story, since synapses would grow in strength without bound. Thus one early question was: How would such modification be stabilized?
Another question was: How is the required information made available at the synaptic junction? The input rate is locally available. The integrated cell response to the inputs from all of the cell's dendrites is not. Thus, in order for the information required for Hebbian modification to be available locally, I conjectured that it must be propagated backwards (by depolarization or spiking in the direction opposite to the usual information flow) from the cell body to each of the synapses, see Figure 1 (Cooper, 1973).

Although such conjectures seemed attractive, there was, in fact, little or no evidence for synaptic modification of any kind at that time. The primary question thus became: Can we find any evidence for synaptic modification? If so, what is its form? Further, what is its cellular and molecular basis - thus the cellular and molecular basis for learning and memory storage?

One way to attack these questions lay in the experimental observation that many cortical neurons are selective. Selectivity is relatively common in the nervous system. Hubel and Wiesel $(1962,1970)$ observed edge detectors in area 17 (V1) of visual cortex of kittens. By the mid 1970s there had already been years of experimentation in visual cortex that had led to two (sometimes controversial) conclusions: In animals with "normal" visual experience, visual cortical neurons are selective and binocular; further, these properties depend on the visual experience of the animal (Blakemore and Cooper, 1970; Hirsch and Spinelli, 1971; Pettigrew and Freeman, 1973; Imbert and Buisseret, 1975).

Thus it seemed that the input-output relations of these neurons could be altered by visual experience - a possible indication of synaptic modification that might be testable experimentally. Could these experience dependent changes in the input-output properties of neurons be attributed to synaptic modification? If so what kind of modification could explain what was seen?

Early models were rate based (von der Malsburg, 1973; Nass and Cooper, 1975; Perez et al., 1975; Cooper et al., 1979). In particular the BCM theory (Bienenstock et al., 1982), created to stabilize Hebbian modification and give the desired neuron selectivity, has diverse consequences that have been shown to be in agreement with observation (Cooper et al., 2004). Essential postulates of BCM are the existence the LTD and LTP regions as well as the sliding modification threshold. These are very simple mathematical requirements but demand rather complicated cell properties. Are they there?

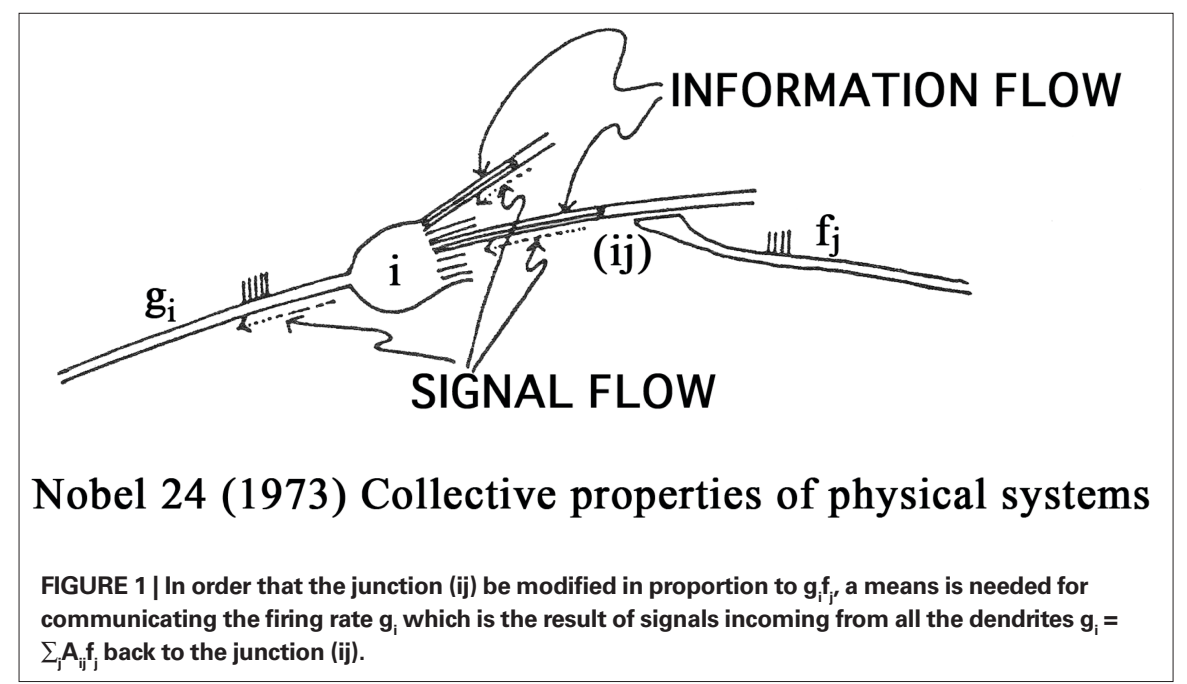


Do neurons really possess these properties? While there was experimental evidence for LTP (Bliss and Lømo, 1973), there was little indication that LTD existed in neocortex or Hippocampus. Such questions were the inspiration for the experiments that led to the first observation of LTD and the sliding modification threshold (Dudek and Bear, 1992; Kirkwood et al., 1996).

The existence of the back propagating action potential was established with the work of (Amitai et al., 1993; Kim and Connors, 1993 and Stuart and Sakmann, 1994); it was further shown that this was required for LTP (Magee and Johnson, 1997). Earlier work (Levy and Steward, 1983) had suggested that timing of the various synaptic inputs played a crucial role in LTD and LTP. But the discovery of STDP gave striking confirmation not only of the existence of the back propagating action potential but of its important role in synaptic modification (Markram et al., 1997; Bi and Poo, 1998). As is attested in many articles in this volume the details of synaptic modification depend exquisitely on the timing of the pre-and postsynaptic spikes.

Among the outstanding questions that confront us at the moment is how to obtain STDP synaptic modification as well as BCM type rate-based theories from more fundamental considerations. Attempts in this direction have been made in several ways: One can try to go from STDP spike models to rate-based models such as BCM. In particular, Gerstner and others have made attempts in this direction (Izhikevich and Desai, 2003; Pfister and Gerstner, 2006). In this approach one is confronted with timing problems. For high frequency inputs, for example, which are the pre and which are the postsynaptic spikes? Other attempts to relate spikes to rates as well as to make connections to BCM have been made (Abarbanel et al., 2002; Appleby and Elliott, 2005; Blais et al., 2009).

Another approach is to attempt from fundamental biophysical models to obtain both STDP as well as BCM rate-based models (Castellani et al., 2001; Shouval et al., 2002a,b). In addition to STDP and presynaptic frequency dependent synaptic plasticity, pairing (voltage clamped) induced synaptic plasticity has been observed. Is there is a single underlying mechanism that can account for different methods of inducing synaptic plasticity?

It had been proposed that a moderate elevation of calcium above baseline produces LTD while a larger elevation produces LTP. (Lynch et al., 1983; Geiger and Singer, 1986; Bear et al., 1987; Lisman, 1989). A calcium control hypothesis that can be derived from lower-level molecular models has been shown to be capable of accounting for the plasticity induced using the various induction mechanisms mentioned above (Shouval et al., 2002b). It required an additional assumption that the back propagating action potential have a wide component. Experimental results suggest that in some dendrites back propagating action potentials are wider than in soma (Magee and Johnson, 1997).

The wide component of the back propagating action potential is also capable of explaining the seeming a-causal behavior of the post-pre spike sequence. The prepost sequence in which the back propagating action potential is presumably initiated at least in part by the pre spike gives the expected LTP. However in the post-pre sequence the post spike might be thought to be produced by spikes arriving at other inputs that should not modify the postpre site. This would occur if we imagine that the post-signal is accompanied by stochastic pre-signals that initiate both LTD and LTP, giving, on average, no change in the synapse.

It has been suggested that the cellular and molecular basis for LTD and LTP involve changes in the number of postsynaptic AMPA receptors as well as phosphorylation and dephosphorylation of specific gluR1 sites on the AMPA receptors (Nayak et al., 1998; Carroll et al., 1999; Shi et al., 1999; Hayashi et al., 2000; Lee et al., 2000). One possibility for the molecular basis of the sliding modification threshold might be variations of the NR2A/2B ratios since changing the NR2A/2B ratio changes the amounts of current that flow through the NMDA receptors. Experience dependent variations of these ratios that have been observed experimentally (Quinlan et al., 1999; Cho and Bear, 2010). It has also been suggested that the $\mathrm{h}$ current may play a role as a basis for the sliding modification threshold (Narayanan et al., 2005).

We thus have a variety of candidates for the cellular and molecular basis for synaptic modification. Among the major remaining questions is to put all of this together in a consistent theory that yields both STDP and rate-based results that is in agreement with observation. And then, of course, this has to be put into networks of neurons to show how we can arrive at brain function, from receptive field formation, learning and memory to mental states including consciousness.

My guess is that we will leave our students a few problems to keep them busy.

\section{REFERENCES}

Abarbanel, H., Huerta, R., and Rabinovich, M. I. (2002). Dynamical model of long-term synaptic plasticity. Proc. Natl. Acad. Sci. U.S.A. 99, 10132-10137.

Amitai, Y., Friedman, A., Connors, B. W., and Gutnick, M. J. (1993). Regenerative activity in apical dendrites of pyramidal cells in neocortex. Cereb. Cortex 3, 26-38.

Anderson, J., Cooper, L. N., Nass, M., Freiberger, W., and Grenender, W. (1972). Some properties of a neural model for memory. AAAS Symposium.

Appleby, P.A., and Elliott, T.(2005).Synaptic and Temporal Ensemble Interpretation of Spike-Timing-Dependent Plasticity. Neural. Comput. 17, 2316-2336.

Bear, M. F., Cooper, L. N., and Ebner, F. F. (1987). A physiological basis for a theory of synapse modification. Science 237, 42-48.

Bi, G. Q., and, Poo, M. M. (1998). Synaptic modifications in cultured hippocampal neurons: dependence on spike timing, synaptic strength, and postsynaptic cell type. J. Neurosci. 18, 10464-10472.

Bienenstock, E. L., Cooper,L. N., and Munro, P.W. (1982). Theory for the development of neuron selectivity: orientation specificity and binocular interaction in visual cortex. J. Neurosci. 2, 32-48.

Blais, B. S., Cooper, L. N., and Shouval, H.Z. (2009). Effect of correlated lateral geniculate nucleus firing rates on predictions for monocular eye closure versus monocular retinal inactivation. Phys. Rev. EStat. Nonlin. Soft Matter Phys. 80, 061915-1-9. (see appendix).

Blakemore, C., and Cooper, G. F. (1970). Development of the brain depends on visual environment. Nature 228, 477-478.

Bliss, T. V. P., and Lømo, T. (1973). Long-lasting potentiation of synaptic transmission in the dentate area of the anaesthetized rabbit following stimulation of the perforant path. J. Physiol. 232, 331-356.

Carroll, R. C., Beattie, E. C., Xia, H., Lüscher, C., Altschuler, Y., Nicoll, R. A., Malenka, R. C., and von Zastrow, M. (1999). Dynamin-dependent endocytosis of ionotropic glutamate receptors. Proc. Natl.Acad. Sci. U.S.A. 96, 14112-14117.

Castellani, G. C., Quinlan, E. M., Cooper, L. N., and Shouval, H. Z. (2001). A biophysical model of bidirectional synaptic plasticity: dependence on AMPA and NMDA receptors. Proc. Natl. Acad. Sci. U.S.A. 98, 12772-12777.

Cho, K. K. A., and Bear, M. F. (2010). Promoting neurological recovery of function via metaplasticity. Future Neurobiol. 5, 21-26.

Cooper, L. N. (1973). "A possible organization of animal memory and learning," in Nobel Symposium on Collective Properties of Physical Systems, eds B. Lundquist and S. Lundquist (New York: Academic Press), 252-264. 
Cooper, L. N., Intrator, N., Blais, B. S., and Shouval, H. Z. (2004). Theory of Cortical Plasticity. Singapore: World Scientific Publishing.

Cooper, L. N., Liberman, F., and Oja, E. (1979). A theory for the acquisition and loss of neuron specificity in visual cortex. Biol. Cybern. 33, 9-28.

Dudek, S. M., and Bear, M.F. (1992). Homosynapticlongterm depression in area CA1 of hippocampus and effects of $N$-methyl-D-aspartate receptor blockade. Proc. Natl. Acad. Sci. U.S.A. 89, 4363-4367.

Geiger, H., and Singer, W. (1986). A possible role of calcium currents in developmental plasticity. Exp. Brain Res. Ser. 14, 256-270.

Hayashi,Y., Shi, S. H., Esteban, J.A., Piccini, A., Poncer, J.C., and Malinow, R. (2000). Driving AMPA receptors into synapses by LTP and CaMKII: requirement for GluR1 and PDZ domain interaction. Science 287, 2262.

Hebb, D. O. (1949). The Organization of Behavior. New York: Wiley.

Hirsch, H. V. B., and Spinelli, D. N. (1971). Modification of the distribution of receptive field orientation in cats by selective visual exposure during development. Exp. Brain Res. 12, 509-527.

Hubel, D. H., and Wiesel, T. N. (1962). Receptive fields, binocular interaction and functional architecture in the cat's visual cortex. J. Physiol. (Lond.) 160 106-154.

Hubel, D. H., and Wiesel, T. N. (1970). The period of susceptibility to the physiological effects of unilateral eye closure in kittens. J. Physiol. (Lond.) 206, 419-436.

Imbert, M., and Buisseret, P. (1975). Receptive field characteristics and plastic properties of visual cortical cells in kittens reared with or without visual experience. Exp. Brain Res. 22, 2-36.

Izhikevich, E., and Desai, N. (2003). Relating STDP to BCM. Neural Comput. 15, 1511-1523.

Kim, H. G., and Connors, B. W. (1993). Apical dendrites of the neocortex: correlation between sodium-, and calcium-dependent spiking, and pyramidal cell morphology. J. Neurosci. 13, 5301-5311.
Kirkwood, A., Rioult, M. G., and Bear, M. F. (1996). Experience-dependent modification of synaptic plasticity in visual cortex. Nature 381, 526-528.

Lee, H. K., Barbarosie, M., Kameyama, K., Bear, M. F., and Huganir, R. (2000). Regulation of distinct AMPA receptor phosphorylation sites during bidirectional synaptic plasticity. Nature 405, 955-959.

Levy,W.B., andSteward,O.(1983).Temporal contiguityrequirements for long-term associative potentiation/depression in the hippocampus. Neuroscience 8, 791-797.

Lin, X. Y., and Glanzman, D. L. (1994). Long-term potentiation of Aplysia sensorimotor synapses in cell culture: regulation by postsynaptic voltage. Proc. Biol. Sci. 255, 113-118.

Lisman, J. A. (1989). A mechanism for the Hebb and the anti-Hebb processes underlying learning and memory. Proc. Natl. Acad. Sci. U.S.A. 86, 9574-9578.

Lynch, G., Larson, J., Kelso, S., Barrionuevo, G., and Schottler, F. (1983). Intracellular injections of EGTA block induction of hippocampal long-term potentiation. Nature 305, 719-721.

Magee, J., and Johnson, D. (1997). A synaptically controlled, associative signal for Hebbian plasticity in hippocampal neurons. Science 275, 209-213.

Markram, H., Lubke, J., Frotscher, M., and Sakmann, B. (1997). Regulation of synaptic efficacy by coincidence of postsynaptic APs and EPSPs. Science 275, 213-215.

Narayanan, R., Brager,D.H., Fan, Y., and Johnston, D. (2005). I_h as a candidate mechanism for sliding the BCM modification threshold. Soc. Neurosci. Abstr. 737.5.

Nass, M., and Cooper, L. N. (1975). A theory for the development of feature detecting cells in visual cortex. Biol. Cybern. 19, 1.

Nayak, A., Zastrow, D., Lickteig, R., Zahniser, N., and Browning, M. (1998). Maintenance of late-phase LTP is accompanied by PKA-dependent increase in AMPA receptor synthesis. Nature 394, 680-683.

Perez, R., Glass, L., and Shlaer, R. J. (1975). Development of specificity in the cat visual cortex. J. Math. Biol. 1, 275-288.
Pettigrew, J. D., and Freeman, R. D. (1973). Visual experience without lines: Effects on developing cortical neurons. Science 182, 599-601.

Pfister, J. P., and Gerstner, W. (2006). Triplets of spikes in a model of spike timing-dependent plasticity. $J$. Neurosci. 26, 9673-9682.

Quinlan, E. M., Philpot, B. D., Huganir, R. L., and Bear, M.F. (1999). Rapid, experience-dependent expression of synaptic NMDA receptors in visual cortex in vivo. Nat. Neurosci. 2, 352-357.

Shi, S. H., Hayashi, Y., Petralia, R. S., Zaman, S. H., Wenthold, R. J., Svoboda, K., and Malinow, R. (1999). Rapid spine delivery and redistribution of AMPA receptors after synaptic NMDA receptor activation. Science 284, 1811.

Shouval, H. Z., Bear, M. F., and Cooper, L. N. (2002a). A unified model of NMDA receptor-dependent bidirectional synaptic plasticity. Proc. Natl. Acad. Sci. U.S.A. 99, 10831-10836.

Shouval, H. Z., Castellani, G. C., Blais, B. S., Yeung, L. C., and Cooper, L. N. (2002b). Converging evidence for a simplified biophysical model of synaptic plasticity. Biol. Cybern. 87, 383-391.

Stuart, G., and Sakmann, B. (1994). Active propagation of somatic action potentials into neocortical pyramidal cell dendrites. Nature 367, 69-72.

von der Malsburg, C. (1973). Self-organization of orientation sensitive cells in the striate cortex. Kybernetik 14, 85-100.

Received: 23 March 2010; accepted: 19May 2010; published online: 07 June 2010.

Citation: Cooper LN (2010) STDP: spiking, timing, rates and beyond Front. Syn. Neurosci. 2:14. doi: 10.3389/ fnsyn.2010.00014

Copyright (c) 2010 Cooper. This is an open-access article subject to an exclusive license agreement between the authors and the Frontiers Research Foundation, which permits unrestricted use, distribution, and reproduction in any medium, provided the original authors and source are credited. 